\title{
Genetics Of Human Origin and Evolution: High-Altitude Adaptations
}

Abigail W. Bigham ${ }^{1}$

1. Department of Anthropology, University of Michigan, Ann Arbor, MI, USA

Corresponding Author:

Abigail W. Bigham

Department of Anthropology

University of Michigan

Ann Arbor, MI, USA

Email: awbigham@umich.edu

Phone: (734) 763-9206

Fax: (734) 763-6077

Word Count: 2,090

(C) 2016. This manuscript version is made available under the Elsevier user license http://www.elsevier.com/open-access/userlicense/1.0/ 


\section{Abstract (116/120):}

High altitude, defined as elevations lying above 2,500 m sea level, challenges human survival and reproduction. This environment provides a natural experimental design wherein specific populations, Andeans, Ethiopians, and Tibetans, have lived in a chronic hypoxia state for millennia. These human groups have overcome the low ambient oxygen tension of high elevation via unique physiologic and genetic adaptations. Genomic studies have identified several genes that underlie high-altitude adaptive phenotypes, many of which are central components of the Hypoxia Inducible Factor (HIF) pathway. Further study of mechanisms governing the adaptive changes responsible for high-altitude adaptation will contribute to our

understanding of the molecular basis of evolutionary change and assist in the functional annotation of the human genome. 


\section{Introduction}

High-altitude hypoxia, experienced at elevations $\geq 2,500$ meters $(\mathrm{m})$ presents numerous challenges to human health, survival, and reproduction as a result of decreased oxygen availability brought on by lowered barometric pressure at high elevations. High-altitude sojourners often suffer from acute mountain sickness whereas residents may experience chronic mountain sickness (CMS). Nonetheless, human's have resided for millennia in three high-altitude zones of the globe: the Qinghai-Tibetan Plateau, the Andean Altiplano, and the Semien Plateau in Ethiopia. Populations from each of these regions have adjusted to the rarified air via unique physiological adaptations that aid in the biological response to cellular hypoxia. Changes in pulmonary function, arterial oxygen saturation $\left(\mathrm{SaO}_{2}\right)$ hemoglobin concentration, and maternal physiology during pregnancy among others have permitted high-altitude natives to thrive in the harsh conditions (Table 1) [1]. Recent research indicates a genetic basis for these physiological differences, highlighting the contribution of genetic adaptation not simply acclimatization in high-altitude adaptive phenotypes [1-3]. Moreover, it has been proposed that high-altitude adaptation is one of the strongest instances of natural selection acting on humans [4]. Thus, these settings afford an unprecedented opportunity to study the effects of selective pressures on human variation and identify the underlying genetic mechanisms governing these populations' physiological adaptations. Here, I discuss what is known about the major genetic adaptations discovered in each of the three human groups. I then address avenues for productive future research.

\section{Genetic adaptations to high-altitude hypoxia}

Emerging genetic data support an evolutionary origin for high-altitude adaptive phenotypes. Furthermore, these data indicate local adaptation via population specific genetic changes wherein different genes have been acted upon by natural selection in each of the three high-altitude human populations. One genetic pathway has been implicated widely in human 
high-altitude adaptation, the Hypoxia Inducible Factor (HIF) pathway. HIF is an evolutionarily ancient oxygen regulator that controls hundreds of downstream genes in response to cellular hypoxia. Andean Quechua and Aymara genomic scans for selection have revealed multiple candidate gene targets for natural selection including at least $40 \mathrm{HIF}$ pathway genes as well as other non-HIF genes [5,6]. From these data, several genomic regions emerge as compelling candidates contributing to Andean altitude-adaptive phenotypes: the HIF genes EGLN1, PRKAA1, NOS2, TGFA, and CXCR4 as well as a region on chromosome 12 falling between 109 and 113 megabase (mb) pairs [5,7]. In total, 47 genes are encoded by the chromosome 12 region and include those involved in cellular housekeeping and immunity. It is important to note that genes in this region have not been previously implicated in oxygen sensing or other altitude adaptive phenotypic pathways. Genome scans performed among high-altitude Argentinian Colla and an intermediate altitude population, Argentinian Calchaquíes, implicate HIF (VEGFB) and non-HIF genes (ELTD1, PRKG1) $[8,9]$. These findings point to the nitric oxide pathway as well as genes involved in cardiac reinforcement as part of the Andean adaptive toolkit. They further highlight that regional populations across the Andes may have experienced population specific genetic changes to hypoxia.

Of these candidate loci, EGLN1, displays compelling overlap with a second high-altitude adapted population, Tibetans $[5,10]$. EGLN1 regulates the HIF transcriptional pathway via decreased hydroxylation of the HIF-1 $\alpha$ subunit (HIF-1 $\alpha)$ during cellular hypoxia [11-13]. This permits HIF1 to continually target downstream genes to maintain cellular oxygen homeostasis. Support for phenotypic consequence of EGLN1 mutations comes from humans who possess loss of function mutations wherein heterozygous individuals display erythrocytosis (elevated hemoglobin concentration) [14,15]. Among Andeans, however, no associations between putative functional alleles at this locus and Andean altitude-adaptive phenotypes, including hemoglobin concentration, have been identified to date [16]. 
Variation near a second candidate locus, PRKAA1, has been linked to fetal growth and birth outcomes among Andeans [17]. Hypoxic stress restricts fetal growth and increases infant morbidity and mortality [18,19]. Indeed, birth weight declines an average of $102 \mathrm{~g}$ per $1000 \mathrm{~m}$ gain in altitude [20]. However, this effect is mitigated in women who are multi-generational residents of altitude. For example, Tibetan and Andean women give birth to larger babies than Han Chinese or European women, respectively, living at the same elevation regardless of socioeconomic status or gestational age [21-23]. Maternal variation at a SNP upstream of PRKAA1 associates with birth weight as well as uterine artery diameter, an intermediate phenotype influencing fetal growth [17]. Furthermore, pointing to a functional link between variation at this site and advantageous Andean phenotypes, SNP genotype is associated with mRNA expression of pathways implicated in the pathophysiology of fetal growth restriction.

In addition to genome wide SNP scans for selection, targeted candidate-gene SNP typing and whole-genome sequencing (WGS) have identified loci contributing to high-altitude Andean phenotypes. One gene in particular, ACE, has been implicated in numerous studies of high-altitude adaptation. ACE is a central component of the renin-angiotensin-aldosterone (RAS) system that is involved in the regulation of cardiovascular homeostasis. The insertion (I) allele of a 47-bp insertion-deletion (I/D) polymorphism contributes to higher resting and exercise $\mathrm{SaO}_{2}$ among Peruvian Quechua [24]. However, the I-allele frequency is as high or higher in several sea-level populations compared to high-altitude groups [25] and compelling evidence of natural selection operating at this locus is lacking. WGS of an Andean CMS cohort identified $A N P 32 D$, an oncogene, and SENP1, an enzymatic regulator of erythropoiesis, as possible targets of natural selection [26]. Functional work performed in drosophila indicated that down regulation of these two genes leads to increased hypoxia tolerance. These data indicate the potential for pharmaceutical intervention in treating CMS by targeting protein products of naturally selected genes. Combined, Andean genomic data that includes candidate gene studies, SNP selection screens, and WGS indicate Andean genetic adaptation to high altitude. 
At this time, it is imperative to integrate genotype with phenotype data by assessing the impact of candidate SNPs on protein function. Only then will we will be able to reveal how genetic changes translate into Andean altitude-adaptive phenotypes.

Among Tibetans, a wealth of data support genetic adaptation to high-altitude. Early studies demonstrated that $\mathrm{SaO}_{2}$ and hemoglobin concentration show significant heritability in this population $[27,28]$. Subsequent genome-wide scans for natural selection identified several key HIF pathway genes involved in the Tibetan pattern of adaptation including EPAS1 and EGLN1 [4,5,10,29-32]. Both genes exhibit compelling evidence of natural selection and variants in these genes contribute to the low hemoglobin concentrations observed among Tibetans [4,10,29]. In particular, two, nonsynonomous EGLN1 SNPs, D4E and C127S, are implicated in functional adaptive consequence [33-35]. In vitro assays indicate that the Tibetan (D4E/C127S) EGLN1 haplotype is defective in its hydroxylation of HIF- $\alpha$, leading to diminished down regulation of the HIF pathway [36]. Tibetans, therefore, may overcome hypoxic stress via a loss of function allele that permits continued activation of the HIF pathway.

Like EGLN1, EPAS1 is a major upstream transcriptional regulator of the HIF pathway. In addition to associations between SNPs in this gene and hemoglobin concentration, recent data suggest that EPAS1 promoter variants may contribute to birth weight among Tibetan newborns [37]. However, the EPAS1 functional allele and its genetic mechanism remain to be determined. Further work identifying the causative mutation(s) contributing to Tibetan high-altitude adaptive phenotypes is necessary to characterize the molecular basis of the allele. Intriguingly, the functional Tibetan EPAS1 allele may be derived from Denisovan, an extinct species of Homo discovered in Denisova Cave, Siberia, or Denisovan-related individuals [38].

Emerging genomic data suggest a role for additional HIF-related genes in the Tibetan adaptive landscape. HMOX2 recently has been proposed as a modifier of hemoglobin metabolism [39]. Other long-term resident populations of the Qinghai-Tibetan plateau (Sherpa and Ayurveda) as well as recent migrants to the area (Mongolians) show patterns of natural 
selection that are consistent with Tibetan adaptation [40-43]. Collectively the Tibetan pattern of genetic adaptation to high altitude indicates a suite of genes in the hypoxia pathway that contribute to the Tibetan pattern of high-altitude adaptation. Furthermore, Tibetan high-altitude adaptive variants have been shown to derive from an ancestral high-altitude population most closely related to modern day Sherpa [44]. Together with the Denisovan data, these findings indicate ancient human and archaic introgression to be important evolutionary sources for adaptive variation.

Ethiopian studies of high-altitude adaptation present a unique opportunity to work with closely related ethnic groups that dwell at both high and low altitude in the same geographic region. Among Ethiopian Amhara and Oromo highlanders, genomic scans have been performed on both SNP and WGS data to identify high-altitude adaptive genes [45-48]. These studies revealed distinct genes from the hypoxia pathway as well as genes in unique pathways of adaptation compared to Andean and Tibetan signatures of selection. Genes involved in the hypoxia response showing evidence of adaptive change among Ethiopians include BHLHE41, ARNT2, and THRB $[46,47]$. A gene-rich region on chromosome 19 containing several genes that are involved in vascular physiology, CXCL17 and PAFAH1B3, or have been linked to hypoxia, LIPE, also exhibits a strong signature of selection [46-48]. Strong signatures of natural selection also have been identified for genes involved in pathogen defense (HLA-DR), angiogenesis (VAV3), and calcium update (CBARA1), as well as alcohol dehydrogenase genes $[45,46]$. Of interest, convergent evolution for alcohol dehydrogenase genes has been demonstrated between Tibetan and Andean populations [7]. This points to the potential of convergent evolution across all three high-altitude adapted human groups and possibly indicates adaptive phenotypes yet to be identified.

Linking genotype to phenotype, the phenotypic consequence of several SNPs on hemoglobin concentration has been demonstrated. Two genes, ARNT2 and THRB, show suggestive relationships with hemoglobin concentration also among Amhara [46] and a region 
on chromosome 1 significantly contributes to hemoglobin levels also among Amhara [45]. Furthermore, SNPs in EGLN1 and EPAS1 with demonstrated hemoglobin associations among Tibetans do not contribute to Ethiopian hemoglobin levels [45]. Together these results suggest a third pattern of genetic adaptation wherein Ethiopian highlanders responded to high altitude stress via a different set of genetic loci.

\section{Future Directions}

Recent advancements in the field of high-altitude genomics have begun to answer longstanding questions in high-altitude and evolutionary biology. It is imperative to move forward with research linking genotype to phenotype by identifying the causal mutation(s) responsible for the observed phenotypic adaptations and functionally characterizing the selected alleles. Substantial progress has been made to understand the functional consequence of EGLN1 variation among Tibetans, but more effort is needed to expand these findings beyond a single locus in one high-altitude population. Until then, genes showing evidence of positive selection will remain putatively adaptive. Functional experimentation will be critical for answering evolutionary questions regarding the mode and tempo of these changes, helping to distinguish whether Andeans, Tibetans, and Ethiopians display convergent/parallel evolution in response to the same environmental pressure, gaining insight into the molecular mechanisms of adaptive change, and determining whether functionally different changes in the same gene lead to distinct altitude-adaptive phenotypes.

Genetic change alone does not fully explain the extent of variation observed in highaltitude adaptive phenotypes. Growth and development under hypoxic conditions also are hypothesized to contribute to physiological adaptations by altering an individual's genetic potential [49]. Andean pulmonary volume and $\mathrm{SaO}_{2}$, for example, appear to be phenotypically plastic and shaped by hypoxia exposure during early development $[50,51]$. A second productive arena for future research is thus the characterization of epigenetic change that occurs during 
growth and development at high-altitude. To date, a single study of epigenetic change has been performed showing significant $\mathrm{CpG}$ methylation differences among high- versus low-altitude Ethiopian Oromo [45]. Identifying epigenetic modifications associated with high-altitude exposure will enable us to mechanistically characterize the effects of growth and development at high altitude on gene expression. This may be particularly fruitful among Andeans, for whom developmental adaptation has been considered the main adaptive strategy [52,53]. Migrant study designs wherein both genetic and epigenetic changes are characterized in long-term residents of high-altitude compared to first and second generation down-migrants of highaltitude ancestry have the power to uncover the genomic and epigenomic architecture that together contribute to altitude-adaptive traits. By harnessing genetic and epigenetic methods to clarify the role of genetic adaptation versus developmental adaptation, altitude studies are poised to dig deeper into the 'omic basis of adaptive phenotypes.

\section{Conclusions}

High altitude is an ideal environment in which to study the evolutionary process as it provides scientists with a natural experiment design using separate, highland populations residing on three continents. These conditions afford an unprecedented opportunity to identify the underlying genetic mechanisms governing adaptive physiology. In addition, determining the mechanisms by which an identical environmental pressure results in similar or distinct genetic adaptations will allow evolutionary geneticists to better understand the molecular basis for convergent human adaptations. Such knowledge is likely to profoundly impact our understanding of the genetic control of biological traits as well as provide deeper insight into patterns of human genetic variation, their origins, and the evolutionary forces that maintain them. Furthermore, such knowledge will be important for identifying genetic factors involved in chronic ischemic diseases such as ischemic heart disease, cerebrovascular disease, and chronic obstructive pulmonary disease. 


\section{Acknowledgements}

This research was supported by funds from the University of Michigan, The National Science Foundation (BCS-1132310), The National Institutes of Health (R33-HL-12075), and the American Association of Physical Anthropologists.

\section{References}

1. Bigham AW, Lee FS: Human high-altitude adaptation: forward genetics meets the HIF pathway. Genes Dev 2014, 28:2189-2204.

2. Simonson TS, McClain DA, Jorde LB, Prchal JT: Genetic determinants of Tibetan highaltitude adaptation. Human genetics 2012, 131:527-533.

3. Beall CM: Genetic changes in Tibet. High Altitude Medicine \& Biology 2011, 12:101-102.

4. Yi X, Liang Y, Huerta-Sanchez E, Jin X, Cuo ZX, Pool JE, Xu X, Jiang H, Vinckenbosch N, Korneliussen TS, et al.: Sequencing of $\mathbf{5 0}$ human exomes reveals adaptation to high altitude. Science 2010, 329:75-78.

5. Bigham A, Bauchet M, Pinto D, Mao X, Akey JM, Mei R, Scherer SW, Julian CG, Wilson MJ, Lopez Herraez $D$, et al.: Identifying signatures of natural selection in Tibetan and Andean populations using dense genome scan data. PLoS genetics 2010, 6.

6. Bigham AW, Mao X, Mei R, Brutsaert T, Wilson MJ, Julian CG, Parra EJ, Akey JM, Moore LG, Shriver MD: Identifying positive selection candidate loci for high-altitude adaptation in Andean populations. Human genomics 2009, 4:79-90.

7. Foll M, Gaggiotti OE, Daub JT, Vatsiou A, Excoffier L: Widespread signals of convergent adaptation to high altitude in Asia and america. Am J Hum Genet 2014, 95:394-407.

${ }^{*} A$ hierarchical Bayesian method is developed and applied to detect convergent evolution among high-altitude Himalayan and Andean populations. The results show that convergent evolution has occurred among populations from these regions and that this method is useful when identifying genes, genomic regions, and pathways under convergent selection.

8. Eichstaedt CA, Antão T, Cardona A, Pagani L, Kivisild T, Mormina M: Genetic and phenotypic differentiation of an Andean intermediate altitude population. Physiological Reports 2015, 3:e12376

9. Eichstaedt CA, Antão T, Pagani L, Cardona A, Kivisild T, Mormina M. The Andean adaptive toolkit to counteract high altitude maladaptation: genome-wide and phenotypic analysis of the Collas. PLoS One 2014, 9:e93314.

10. Simonson TS, Yang YZ, Huff CD, Yun HX, Qin G, Witherspoon DJ, Bai ZZ, Lorenzo FR, Xing JC, Jorde LB, et al.: Genetic Evidence for High-Altitude Adaptation in Tibet. Science 2010, 329:72-75.

11. Ivan M, Kondo K, Yang H, Kim W, Valiando J, Ohh M, Salic A, Asara JM, Lane WS, Kaelin WG, Jr.: HIFalpha targeted for VHL-mediated destruction by proline hydroxylation: implications for 02 sensing. Science 2001, 292:464-468. 
12. Hirsila M, Koivunen P, Gunzler V, Kivirikko KI, Myllyharju J: Characterization of the human prolyl 4-hydroxylases that modify the hypoxia-inducible factor. The Journal of biological chemistry 2003, 278:30772-30780.

13. Epstein AC, Gleadle JM, McNeill LA, Hewitson KS, O'Rourke J, Mole DR, Mukherji M, Metzen E, Wilson MI, Dhanda A, et al.: C. elegans EGL-9 and mammalian homologs define a family of dioxygenases that regulate HIF by prolyl hydroxylation. Cell 2001, 107:43-54.

14. Percy MJ, Zhao Q, Flores A, Harrison C, Lappin TR, Maxwell PH, McMullin MF, Lee FS: A family with erythrocytosis establishes a role for prolyl hydroxylase domain protein 2 in oxygen homeostasis. Proceedings of the National Academy of Sciences of the United States of America 2006, 103:654-659.

15. Lee FS, Percy MJ: The HIF pathway and erythrocytosis. Annu Rev Pathol 2011, 6:165-192.

16. Bigham AW, Wilson MJ, Julian CG, Kiyamu M, Vargas E, Leon-Velarde F, Rivera-Chira M, Rodriquez C, Browne VA, Parra E, et al.: Andean and Tibetan patterns of adaptation to high altitude. American journal of human biology : the official journal of the Human Biology Council 2013, 25:190-197.

17. Bigham AW, Julian CG, Wilson MJ, Vargas E, Browne VA, Shriver MD, Moore LG: Maternal PRKAA1 and EDNRA genotypes are associated with birth weight, and PRKAA1 with uterine artery diameter and metabolic homeostasis at high altitude. Physiological Genomics 2014, 46:687-97

18. Keyes LE, Armaza JF, Niermeyer S, Vargas E, Young DA, Moore LG: Intrauterine growth restriction, preeclampsia, and intrauterine mortality at high altitude in Bolivia. Pediatric research 2003, 54:20-25.

19. Mccullough RE, Reeves JT, Liljegren RL: Fetal Growth Retardation and Increased Infant-Mortality at High Altitude. Obstetrics and Gynecological Survey 1977, 32:596598.

20. Jensen GM, Moore LG: The effect of high altitude and other risk factors on birthweight: Independent or interactive effects? American Journal of Public Health 1997, 87:1003-1007.

21. Moore LG, Young D, McCullough RE, Droma T, Zamudio S: Tibetan protection from intrauterine growth restriction (IUGR) and reproductive loss at high altitude. American Journal of Human Biology 2001, 13:635-644.

22. Julian CG, Vargas E, Armaza JF, Wilson MJ, Niermeyer S, Moore LG: High-altitude ancestry protects against hypoxia-associated reductions in fetal growth. Archives of Disease in Childhood-Fetal and Neonatal Edition 2007, 92:372-377.

23. Zamudio S, Droma T, Norkyel KY, Acharya G, Zamudio JA, Niermeyer SN, Moore LG: Protection from Intrauterine Growth-Retardation in Tibetans at High-Altitude. American journal of physical anthropology 1993, 91:215-224.

24. Bigham AW, Kiyamu M, Leon-Velarde F, Parra EJ, Rivera-Ch M, Shriver MD, Brutsaert TD: Angiotensin-converting enzyme genotype and arterial oxygen saturation at high altitude in Peruvian Quechua. High Altitude Medicine \& Biology 2008, 9:167-178.

25. Qadar Pasha MA, Khan AP, Kumar R, Grover SK, Ram RB, Norboo T, Srivastava KK, Selvamurthy W, Brahmachari SK: Angiotensin converting enzyme insertion allele in relation to high altitude adaptation. Ann Hum Genet 2001, 65:531-536.

26. Zhou D, Udpa N, Ronen R, Stobdan T, Liang J, Appenzeller O, Zhao HW, Yin Y, Du Y, Guo $\mathrm{L}$, et al.: Whole-genome sequencing uncovers the genetic basis of chronic mountain sickness in Andean highlanders. American journal of human genetics 2013, 93:452-462. 
27. Beall CM, Blangero J, Williams-Blangero S, Goldstein MC: Major gene for percent of oxygen saturation of arterial hemoglobin in Tibetan highlanders. American journal of physical anthropology 1994, 95:271-276.

28. Beall CM, Brittenham GM, Strohl KP, Blangero J, Williams-Blangero S, Goldstein MC, Decker MJ, Vargas E, Villena M, Soria R, et al.: Hemoglobin concentration of highaltitude Tibetans and Bolivian Aymara. American journal of physical anthropology 1998, 106:385-400.

29. Beall CM, Cavalleri GL, Deng L, Elston RC, Gao Y, Knight J, Li C, Li JC, Liang Y, McCormack M, et al.: Natural selection on EPAS1 (HIF2alpha) associated with low hemoglobin concentration in Tibetan highlanders. Proceedings of the National Academy of Sciences of the United States of America 2010, 107:11459-11464.

30. Peng Y, Yang Z, Zhang H, Cui C, Qi X, Luo X, Tao X, Wu T, Ouzhuluobu, Basang, et al.: Genetic variations in Tibetan populations and high-altitude adaptation at the Himalayas. Molecular biology and evolution 2011, 28:1075-1081.

31. Xu S, Li S, Yang Y, Tan J, Lou H, Jin W, Yang L, Pan X, Wang J, Shen Y, et al.: A genome-wide search for signals of high-altitude adaptation in Tibetans. Molecular biology and evolution 2011, 28:1003-1011.

32. Wang B, Zhang YB, Zhang F, Lin H, Wang X, Wan N, Ye Z, Weng H, Zhang L, Li X, et al.: On the origin of Tibetans and their genetic basis in adapting high-altitude environments. PloS one 2011, 6:e17002.

33. Lorenzo FR, Huff C, Myllymaki M, Olenchock B, Swierczek S, Tashi T, Gordeuk V, Wuren T, Ri-Li G, McClain DA, et al.: A genetic mechanism for Tibetan high-altitude adaptation. Nature genetics 2014, 46:951-956.

${ }^{* *}$ Tibetans harbor two nonsynonmous changes in EGLN1 gene, D4E and C127S, that are markedly enriched in this population and appeared together roughly 8,000 years ago. Together, these changes result in a Tibetan variant protein that the authors suggest is a gain-of-function mutation. These results shed light on the molecular mechanisms of Tibetan adaptation to high altitude.

34. Xiang K, Ouzhuluobu, Peng Y, Yang Z, Zhang X, Cui C, Zhang H, Li M, Zhang Y, Bianba, et al.: Identification of a Tibetan-specific mutation in the hypoxic gene EGLN1 and its contribution to high-altitude adaptation. Molecular biology and evolution 2013, 30:1889-1898.

35. Petousi N, Croft QP, Cavalleri GL, Cheng HY, Formenti F, Ishida K, Lunn D, McCormack $\mathrm{M}$, Shianna KV, Talbot NP, et al.: Tibetans living at sea level have a hyporesponsive hypoxia-inducible factor system and blunted physiological responses to hypoxia. Journal of Applied Physiology 2014, 116:893-904.

36. Song D, Li LS, Arsenault PR, Tan Q, Bigham AW, Heaton-Johnson KJ, Master SR, Lee FS: Defective Tibetan PHD2 Binding to p23 Links High Altitude Adaptation to Altered Oxygen Sensing. The Journal of biological chemistry 2014, 289:14656-14665.

** The dominant Tibetan EGLN1 haplotype at sites D4E and C127S reduces the interaction of the EGLN1 protein with p23, an HSP cochaperone important in HIF- $\alpha$ hydroxylation. This suggests that the adaptive Tibetan EGLN1 allele results in a loss of function that leads to impaired downregulation of the HIF pathway to facilitate adaptation to high altitude.

37. Xu XH, Huang XW, Qun L, Li YN, Wang Y, Liu C, Ma Y, Liu QM, Sun K, Qian F, et al.: Two functional loci in the promoter of EPAS1 gene involved in high-altitude adaptation of Tibetans. Sci Rep 2014, 4:7465.

38. Huerta-Sanchez E, Jin X, Asan, Bianba Z, Peter BM, Vinckenbosch N, Liang Y, Yi X, He $\mathrm{M}$, Somel $\mathrm{M}$, et al.: Altitude adaptation in Tibetans caused by introgression of Denisovan-like DNA. Nature 2014, 512:194-197. 
**The dominant Tibetan EPAS1 haplotype was introgressed from Denisovan or Denisovan-derived individuals. This demonstrates the importance of adaptive introgressed variation in the genetic architecture of Tibetan genetic adaptation.

39. Yang D, Peng Y, Ouzhuluobu, Bianbazhuoma, Cui C, Bianba, Wang L, Xiang K, He Y, Zhang $\mathrm{H}$, et al.: HMOX2 Functions as a Modifier Gene for High-Altitude Adaptation in Tibetans. Hum Mutat 2016, 37:216-223.

40. Hanaoka M, Droma Y, Basnyat B, Ito M, Kobayashi N, Katsuyama Y, Kubo K, Ota M: Genetic variants in EPAS1 contribute to adaptation to high-altitude hypoxia in Sherpas. PloS one 2012, 7:e50566.

41. Kang L, Zheng HX, Chen F, Yan S, Liu K, Qin Z, Liu L, Zhao Z, Li L, Wang X, et al.: mtDNA lineage expansions in Sherpa population suggest adaptive evolution in Tibetan highlands. Molecular biology and evolution 2013, 30:2579-2587.

42. Aggarwal S, Negi S, Jha P, Singh PK, Stobdan T, Pasha MA, Ghosh S, Agrawal A, Prasher B, Mukerji M: EGLN1 involvement in high-altitude adaptation revealed through genetic analysis of extreme constitution types defined in Ayurveda. Proceedings of the National Academy of Sciences of the United States of America 2010, 107:18961-18966.

43. Xing J, Wuren T, Simonson TS, Watkins WS, Witherspoon DJ, Wu W, Qin G, Huff CD, Jorde $L B, G e$ RL: Genomic analysis of natural selection and phenotypic variation in high-altitude mongolians. PLOS genetics 2013, 9:e1003634.

44. Jeong C, Alkorta-Aranburu G, Basnyat B, Neupane M, Witonsky DB, Pritchard JK, Beall CM, Di Rienzo A: Admixture facilitates genetic adaptations to high altitude in Tibet. Nat Commun 2014, 5:3281.

*This research identifies a novel mechanism, admixture-mediated adaptation, of adaptation

The modern Tibetan population is an admixed population comprised of ancestral groups related to present day Nepalese Sherpa and Han Chinese. The results indicate that admixture-mediated adaptation has been an important mechanism beyond selection through which Tibetans have adapted to hypoxia.

45. Alkorta-Aranburu G, Beall CM, Witonsky DB, Gebremedhin A, Pritchard JK, Di Rienzo A: The genetic architecture of adaptations to high altitude in Ethiopia. PLOS Genet 2012, 8:e1003110.

46. Scheinfeldt LB, Soi S, Thompson S, Ranciaro A, Woldemeskel D, Beggs W, Lambert C, Jarvis JP, Abate D, Belay G, et al.: Genetic adaptation to high altitude in the Ethiopian highlands. Genome biology 2012, 13:R1.

47. Huerta-Sanchez E, Degiorgio M, Pagani L, Tarekegn A, Ekong R, Antao T, Cardona A, Montgomery HE, Cavalleri GL, Robbins PA, et al.: Genetic signatures reveal highaltitude adaptation in a set of ethiopian populations. Molecular biology and evolution 2013, 30:1877-1888.

48. Udpa N, Ronen R, Zhou D, Liang J, Stobdan T, Appenzeller O, Yin Y, Du Y, Guo L, Cao $\mathrm{R}$, et al.: Whole genome sequencing of Ethiopian highlanders reveals conserved hypoxia tolerance genes. Genome biology 2014, 15:R36.

49. Frisancho AR: Developmental adaptation to high altitude hypoxia. Int J Biometeorol 1977, 21:135-146.

50. Kiyamu M, Bigham A, Parra E, Leon-Velarde F, Rivera-Chira M, Brutsaert TD: Developmental and genetic components explain enhanced pulmonary volumes of female Peruvian Quechua. American journal of physical anthropology 2012, 148:534542.

51. Kiyamu M, Leon-Velarde F, Rivera-Chira M, Elias G, Brutsaert TD: Developmental Effects Determine Submaximal Arterial Oxygen Saturation in Peruvian Quechua. High Alt Med Biol 2015, 16:138-146. 
*Elevated arterial oxygen saturation has been previously identified as an Andean highaltitude adaptive trait. This study demonstrates compelling evidence for a developmental component to their adaptive higher arterial oxygen saturation phenotype.

52. Brutsaert TD: Genetic and environmental adaptation in high altitude natives. Conceptual, methodological, and statistical concerns. Adv Exp Med Biol 2001, 502:133-151.

53. Frisancho AR, Frisancho HG, Albalak R, Villain M, Vargas E, Soria R: Developmental, genetic, and environmental components of lung volumes at high altitude. American Journal of Human Biology 1997, 9:191-203.

\section{Tables}

Table 1. Physiologic adaptations to high-altitude hypoxia among high-altitude populations*

\section{Phenotype}

Resting Ventilation

Hypoxic Ventilatory Response

Arterial Oxygen Saturation

Hemoglobin Concentration

Birth Weight
Andean

No increase

Blunted (low)

Elevated

Elevated

Elevated

\section{Tibetan}

$50 \%$ higher

Similar to sea-level

No increase

Lowered

Elevated
Ethiopian

NR

NR

Elevated

Minimal Increase

NR

*Adapted from [1].

\section{Figure Legends}

\section{Figure 1. HIF pathway candidate genes for human adaptation to high altitude}

The three geographic regions where humans have adapted to high altitude are highlighted in blue and include the Andean Altiplano, Semien Plateau, and the Tibetan Plateau. HIF pathway candidate genes for each population are listed in each of the population boxes. Genes listed in black are unique to the particular population, blue are shared between all three, green are shared between Tibetan and Andean, red are shared between Andean and Ethiopian, and purple are shared between Tibetan and Ethiopian. 
Figure 1

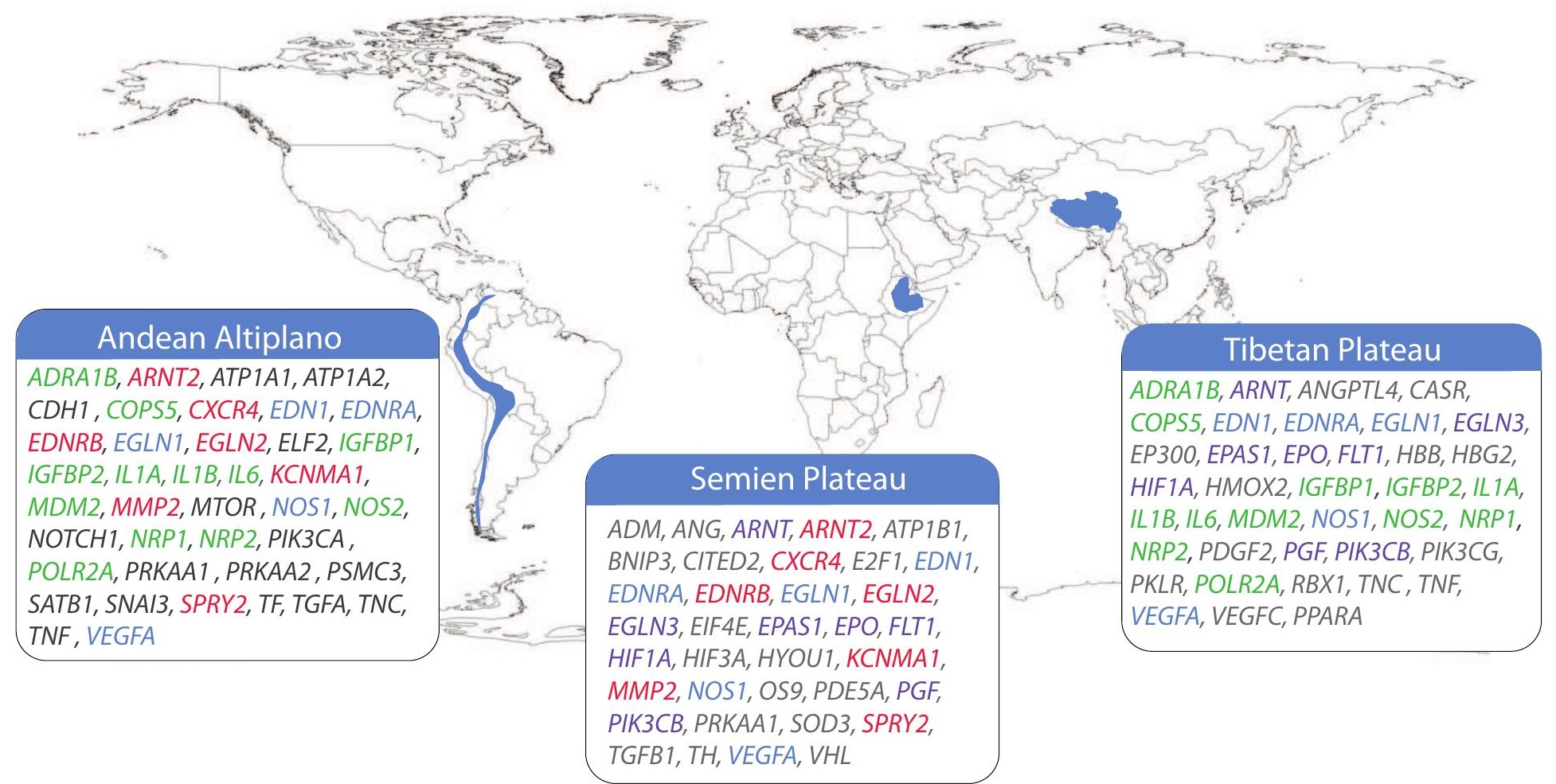

ADRA1B, ARNT2, ATP1A1, ATP1A2,

CDH1, COPS5, CXCR4, EDN1, EDNRA, EDNRB, EGLN1, EGLN2, ELF2, IGFBP1, IGFBP2, IL1A, IL1B, IL6, KCNMA1, MDM2, MMP2, MTOR, NOS1, NOS2, NOTCH1, NRP1, NRP2, PIK3CA , TNF, VEGFA MMP2, NOS1, OS9, PDE5A, PGF,

\section{Tibetan Plateau} ADRA1B, ARNT, ANGPTL4, CASR, COPS5, EDN1, EDNRA, EGLN1, EGLN3, EP300, EPAS1, EPO, FLT1, HBB, HBG2, GFBP2, ILTA, ILT, IL6, MDM2, NOS1, NOS2, NRP 1, NRP2, PDGF2, PGF, PIK3CB, PIK3CG, PKLR, POLR2A, RBX1, TNC, TNF, VEGFA, VEGFC, PPARA

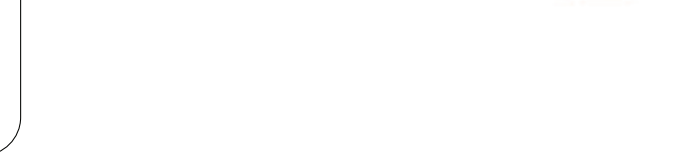

